\title{
Short interfering RNA directed against the E2F-1 gene suppressing gastric cancer progression in vitro
}

\author{
YUBO XIE $^{1,2}$, YONGSHUO YIN ${ }^{1}$, LEI LI $^{1}$, YULIN MA ${ }^{1}$ and QIANG XIAO $^{1}$ \\ Departments of ${ }^{1}$ Surgery, and ${ }^{2}$ Anesthesiology, The First Affiliated Hospital, \\ Guangxi Medical University, Nanning, Guangxi, P.R. China
}

Received December 22, 2008; Accepted February 18, 2009

DOI: 10.3892/or_00000360

\begin{abstract}
Gastric cancer is the third most common cancer in China. The sustained overexpression of E2F-1 is a characteristic feature of gastric cancer. RNA interference (RNAi), which has been proven to be a powerful tool for suppressing gene expression, may provide a promising way forward in gastric cancer therapy. In this study, we constructed the recombinant Psilencer 4.1- E2F-1 siRNA plasmids and transfected them into gastric cancer MGC-803 cells in vitro. Our data demonstrated that E2F-1 siRNA led to inhibition of endogenous E2F-1 mRNA and protein expression as determined by real-time quantitative RT-PCR and Western blotting. Furthermore, simultaneous silencing of E2F-1 resulted in a reduction of tumor cell proliferation activity and a higher percentage of apoptotic cells. The inhibition of migration and invasion potential of tumor cells was investigated in vitro. In summary, siRNA targeting of E2F-1 can effectively inhibit gastric cancer progression and may be used as a potent therapy.
\end{abstract}

\section{Introduction}

Gastric cancer is one of the most frequent cancers and one of the most frequent causes of cancer-related mortality in China, with an incidence of 0.4 million new cases and 0.3 million deaths annually, and it ranks the third most common cancer in China (1). Although diagnostic and surgical techniques for treatment of gastric cancer have advanced in recent years, post-surgery survival rates have not improved in the last decade. The overall 5-year survival rate generally remains less than $30 \%$, even after three-field lymph node dissection or combined chemotherapy and radiotherapy.

Tumor cell proliferation, migration and invasion which are processes controlled by a very complex cell cycle machinery are main causes of death in patients with gastric cancer.

Correspondence to: Professor Qiang Xiao, Department of Surgery, The First affiliated Hospital, Guangxi Medical University, No. 22 Shuang-yong Road, Nanning, Guangxi 530021, P.R. China E-mail: xiaoqiang20050@yahoo.com.cn

Key words: small interfering RNA, E2F-1, gastric cancer, MGC-803 cells
Multiple components such as cyclins, cyclin dependent kinases (Cdks), Cdk inhibitors, Rb, and E2Fs are implicated in the transition from one cell cycle phase to another. For example, D-type cyclins bind and activate Cdk4/Cdk6, which phosphorylate the $\mathrm{Rb}$ gene product $\mathrm{pRb}$, whereas $\mathrm{Cdk}$ inhibitors such as p21 and p16 can inhibit the function of Cdks. As a result of phosphorylation of pRb, E2F is released from the $\mathrm{pRb}-\mathrm{E} 2 \mathrm{~F}$ complex. The E2F is an active transcription factor that promotes the transcription of the genes required for DNA synthesis and drives cells from the G1 phase into the S phase (2-4).

Dysregulation of the normal cell cycle-regulatory machinery is integral to the neoplastic process, and there is now compelling evidence implicating loss of cell cycle control in the development and progression of most human cancers (5). The transcription factor E2F-1 is the best-known ultimate transcription factor activated in the cyclin-Cdk-Rb pathway. E2F-1 can regulate progression through checkpoints in the cell cycle and has a dual role in cancer development with the capacity to act as both a tumor suppressor gene and an oncogene (6). E2F-1 enhances proliferation in gastrointestinal carcinomas while conversely stimulating apoptosis in bladder cancers independent of $\mathrm{pRb}$ expression (7-9). Overexpression of E2F-1 in head and neck squamous carcinoma cell (HNSCC) lines has the capacity to stimulate cell cycle reentry but is also associated with increased invasiveness, suggesting a role in metastasis (10).

The RNA interference (RNAi) means the use of doublestranded RNA (dsRNA) to target specific mRNAs for degradation, thereby silencing their expression. RNAi is one manifestation of a broad class of RNA silencing phenomena that are found in plants, animals and fungi. The discovery of RNAi has changed our understanding of how cells guard their genomes, led to the development of new strategies for blocking gene function, and may yet yield RNA-based drugs to treat human disease (11). Biochemical and molecular evidence suggests that short interfering RNAs (siRNAs) are generated in cells by endonucleolytic cleavage of exogenous dsRNA. The siRNAs are then thought to incorporate into a protein-RNA complex, the RNA-induced silencing complex (RISC), where they serve to guide a nuclease to the target mRNA (12).

To study whether E2F-1 plays a role in tumor progression, we transfected gastric cancer MGC-803 cells with E2F-1 siRNA to inhibit E2F-1 expression and investigated the 
Table I. The base sequence of primers and probes for real-time quantitative RT-PCR.

\begin{tabular}{llll}
\hline Gene & Primer & Base sequence & PCR product (bp) \\
\hline E2F-1 & Forward & 5'-ACCCTGCAGAGCAGATGGTT-3' & 100 \\
& Reverse & 5'-TTTGCTCTTAAGGGAGATCTGAA-3' & \\
B-actin & Forward & 5'-AACTCCATCATGAAGTGTGA-3' & 247 \\
& Reverse & 5'-ACTCCTGCTTGCTGATCCAC-3' & \\
\hline
\end{tabular}

ability of proliferation, migration and invasion suppressed by E2F-1 siRNA in gastric cancer cells.

\section{Materials and methods}

Cell culture. The human gastric carcinoma MGC-803 cells were cultured in Dulbecco's modified Eagle's medium (DMEM) (Invitrogen, Gaithersburg, MD, USA). All media were supplemented with $10 \% \mathrm{FBS}$, penicillin $(100 \mathrm{U} / \mathrm{ml})$, and streptomycin $(100 \mu \mathrm{g} / \mathrm{ml})$. The cells were cultured in an incubator at $5 \% \mathrm{CO}_{2}$ and $37^{\circ} \mathrm{C}$, with medium changes every 3 days.

Construction and transfection of the siRNA plasmid expression vector. Double chains of oligonucleotide with complementary sequences that can code siRNA were obtained from Genesil Biotechnology Company (China). There were two reversed repeated sequences with 19 inserted sequences (CTGACTCTGCCACCATAGT, ACTATGGTGGCAGA GTCAG) in this complementary sequence, with BamHI and HindIII in both sides for the ligation to Psilencer 4.1 vector. The siRNA targeting site of the transcripted product was nucleotides 124-1437 of E2F-1 mRNA (GeneBank No. NM-005225). The negative control was the siRNA sequence from the transcripted product of double-chain oligonucleotide with no homology to any human gene sequence.

After the ligation, the plasmid was transformed into Escherichia coli TOP10 cells, and then planted on solid LB medium. Ampicillin-resistant colonies were cultured at $37^{\circ} \mathrm{C}$ overnight in a rocking bed. The recombinant E2F-1 siRNA plasmid was prepared, and the sequences were verified by electrophoresis of the digested product. The inserted siRNA was $55 \mathrm{bp}$. MGC-803 cells $\left(1 \times 10^{5}\right)$ were inoculated into a 6-well plate and transfected with Psilencer 4.1- E2F-1(+) or Psilencer 4.1- E2F-1(-) recombination plasmids when the confluence was $90 \%$. Forty-eight hours after transfection, MGC-803 cells were diluted to 1:10 for passage, and cultured for at least 2 weeks in medium with G418. The cells were divided into 3 groups: MGC-803/Silence(+) cells, MGC-803/ Silence(-) cells and MGC-803 cells.

Real-time quantitative RT-PCR. Total RNAs were extracted from positive cell clone using TRIzol Reagent (Invitrogen). Neo gene segments were amplified and verified by real-time RT-PCR. Complementary deoxyribonucleic acids (cDNAs) were reverse-transcribed from $2 \mu \mathrm{g}$ of total RNA. Table I shows the PCR primer sequences used in this study. The products of PCR were checked by agarose gel electro- phoresis, and the abundance of each mRNA was detected and normalized to that of glyceraldehyde-3-phosphate dehydrogenase (ß-actin) mRNA.

Western blot analysis. Cell lysates were prepared in a buffer containing $0.1 \mathrm{M} \mathrm{NaCl}, 0.01 \mathrm{M}$ Tris-Cl (pH 7.6), $0.001 \mathrm{M}$ EDTA (pH 8.0), $1 \mu \mathrm{g} / \mathrm{ml}$ aprotinin, $100 \mu \mathrm{g} / \mathrm{ml} \mathrm{PMSF}$, and $1 \%(\mathrm{v} / \mathrm{v})$ NP40. After protein quantitation using the Lowery protein assay, equal amounts of proteins were separated by SDS-PAGE and blotted onto nitrocellulose membranes by the semi-dry blotting method using a three buffer system. The membrane was blocked with 5\%(w/v) BSA in PBST (PBS, $\mathrm{pH} 7.5$, containing $0.1 \%$ Tween-20) and incubated with a 1:500 dilution of primary antibody (anti-E2F-1) (Santa Cruz Biotechnology Inc., Santa Cruz, CA, USA) overnight at $4^{\circ} \mathrm{C}$. The membrane was then washed with PBST and incubated with a peroxidase-conjugated secondary antibody (1:1000) (Santa Cruz Biotechnology) for $1 \mathrm{~h}$. Specific antibody binding was detected using a chemiluminescence detection system (Pierce, USA), according to the manufacturer's recommendations. Western blot film was scanned, and the net intensities of the bands were quantified using Image-QuanT software (Molecular Dynamics, Sunnyvale, CA, USA). After development, the membrane was stripped and reprobed with antibody against $\beta$-actin (1:1000) (Santa Cruz Biotechnology) to confirm equal sample loading.

Cell proliferation and survival assay. The viability and proliferation of MGC-803 cells were determined by MTS assay using a CellTiter 96 AQueous assay system (Promega, Madison, WI, USA), according to the manufacturer's instructions. The assay measures the dehydrogenase enzyme activity in metabolically active tumor cells, as reflected by the conversion of MTS to formazan, which is soluble in tissue culture medium and was detected by absorbance at $490 \mathrm{~nm}$. The production of formazan is proportional to the number of living cells, with the intensity of the produced color serving as an indicator of the cell viability. Briefly, the MGC-803 cells were plated at $1 \times 10^{5}$ cells per well in 96well plates and cultured for $72 \mathrm{~h}$. MTS mixed with medium without serum was added to the cell cultures after removing the old medium. The plates were incubated at $37^{\circ} \mathrm{C}$ for $4 \mathrm{~h}$, and the absorbance (A) at $490 \mathrm{~nm}$ was determined using a 96-well Opsys MR ${ }^{\mathrm{TM}}$ microplate reader (ThermoLab systems, Chantilly, VA, USA) and Revelation ${ }^{\mathrm{TM}}$ QuickLink software. The blank control wells with medium only were set as zero absorbance. The percentage of cell survival was calculated using the background-corrected absorbance: \% cell viability 
$=100 \times \mathrm{A}$ of experimental well/A of untreated control well. All experiments were performed at least three times, and representative data are presented in Fig. 3.

Colony formation assay. Cell suspensions from each group were diluted in DEME with $10 \%$ FBS, and immediately replated in 6-well plates at a density of 20 cells $/ \mathrm{cm}^{2}$. The plates were incubated until cells in control wells formed sufficiently large colonies. After that, the colonies were fixed with $6 \%$ glutaraldehyde and stained with $0.5 \%$ crystal violet. The plates were photographed and their digital images were analyzed manually to known the colony number.

Cell cycle analysis by flow cytometry. For cell cycle analysis, MGC-803 cells $\left(1 \times 10^{6}\right)$ were washed twice with ice-cold PBS, treated with trypsin, and then fixed in $70 \%$ cold ethanol at $4{ }^{\circ} \mathrm{C}$ for $30 \mathrm{~min}$. The cell pellet was incubated in a solution containing $50 \mathrm{ng} / \mathrm{ml}$ propidium iodide, $0.2 \mathrm{mg} / \mathrm{ml}$ RNase, and $0.1 \%$ Triton $\mathrm{X}-100$ at room temperature for $30 \mathrm{~min}$, and then analyzed by flow cytometry using a FACscan (Becton-Dickinson, Mountain View, CA, USA). The data were analyzed with the MultiCycle for Windows (Phoenix Flow Systems, San Diego, USA).

Apoptosis assay by flow cytometry. Apoptotic cells were determined using Annexin V-FITC Apoptosis Detection Kit (Jingmei Biotech Co., Shenzhen, China) and an EPICS XLMCL flow cytometry (Becton-Dickinson) according to the manufacturer's instructions. Briefly, $1 \times 10^{6}$ cells were stained with Annexin V/FITC for $30 \mathrm{~min}$ at $4^{\circ} \mathrm{C}$ in the dark and then propidium iodide for $10 \mathrm{~min}$ before flow cytometric analyses.

Wound healing assay. The cells were cultured to confluence on 6-well plates. A central linear wound was made with a $200 \mu 1$ sterile pipet tip. Media were changed gently to remove any floating cells. Phase micrographs of the wound cultures were taken at $0,24,36$ and $48 \mathrm{~h}$. The photographs were analyzed by measuring the distance from the wound edge of the cell sheet to the original wound site. Migration activity was calculated as the mean distance between edges of three points in 12 fields per well. Relative motility = (mean original distance - mean distance at a time point)/mean original distance $\mathrm{x} 100 \%$. Each test group was assayed in triplicate.

Cell invasion assay. Cell invasion was assessed using Transwell chambers (6.5 mm; Corning, New York, USA) with serafree DMEM $50 \mu \mathrm{l}$ containing $1 \mu \mathrm{g} / \mu \mathrm{l}$ Matrigel (Deparment of Biology, Beijing University, China) in upper chamber. The lower chamber was filled with DMEM $50 \mu$ l containing $0.1 \mu \mathrm{g} / \mu \mathrm{g}$ fibronectin (Beijing University). Cells $\left(1 \times 10^{5}\right)$ were suspended with $100 \mu \mathrm{l}$ DMEM with $1 \%$ fetal calf serum and plated into the upper chamber. PBS (5\%) $500 \mu 1$ was added in lower chamber. After $24 \mathrm{~h}$ incubation at $5 \%$ $\mathrm{CO}_{2}$ and $37^{\circ} \mathrm{C}$, the number of cells with Giemsa staining on the undersurface of the polycarbonate membranes was scored visually in five random fields at x400 magnification using a light microscope.

Statistical analysis. Data are expressed as mean \pm standard error of the mean (SEM) analyzed by SPSS 13.0 (SPSS Inc.,

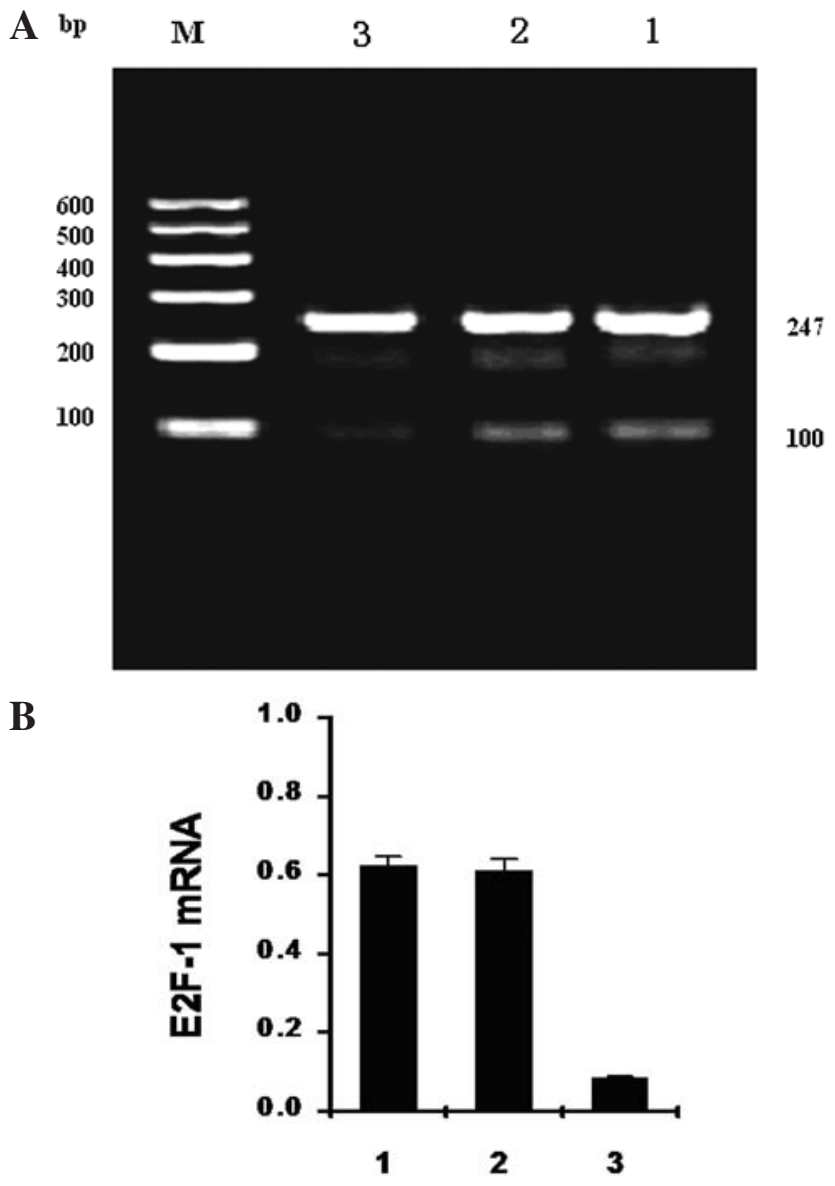

Figure 1. (A) The band of E2F-1 mRNA checked by real-time quantitative RT-PCR. Lane 1, MGC-803 cells; lane 2, MGC-803/Silence(-) cells; lane 3, MGC-803/Silence(+) cells. M, 600 bp marker. (B) The expression of E2F-1 mRNA in MGC-803/Silence(+) cells was strongly suppressed.

Chicago, IL, USA) and Origin 7.5 software programs (OriginLab Co., Northampton, MA, USA). Student's t-test was used to measure statistical significance between two treatment groups. Multiple comparisons were performed with a one-way analysis of variance (ANOVA). Data were considered significant at $\mathrm{P}<0.05$.

\section{Results}

Construction and verification of E2F-1 siRNA plasmid. Recombinant Psilencer 4.1- E2F-1(+) and Psilencer 4.1E2F-1(-) sequences were verified by electrophoresis. The inserted siRNA was 55 bp with digestion sites BamHI and HindIII on both sides, consistent with the designed series. This confirmed that the construction of the E2F-1 siRNA expression plasmid was successful.

Inhibitory effect of the expression of E2F-1 mRNA and protein. The transfection of Psilencer 4.1- E2F-1(+) plasmid into MGC-803 cells led to remarkable inhibition of E2F-1 mRNA and protein expression. Densitometric analysis showed that E2F-1 mRNA and protein in MGC-803/Silence(+) cells were about 6- and 5-fold lower than those of two control groups, respectively $(\mathrm{P}<0.05)$, while no difference was found between MGC-803/Silence(-) cells and MGC-803 cells ( $\mathrm{P}>0.05)$ (Figs. 1 and 2). 
A
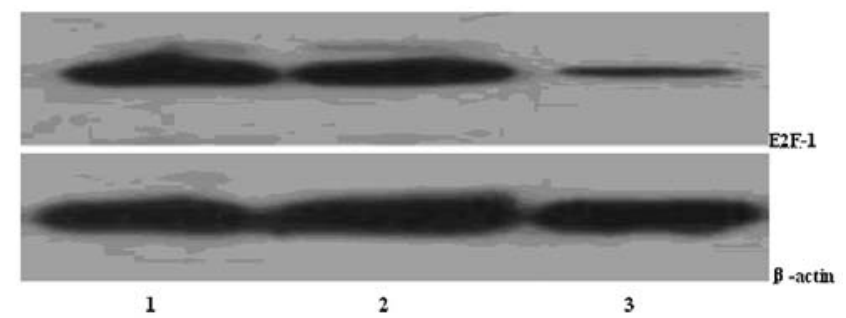

B

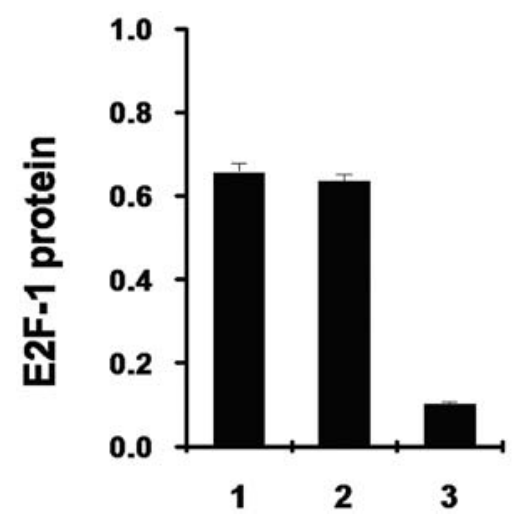

Figure 2. (A) The expression of E2F-1 protein in cells by Western blot analysis. Lane 1, MGC-803 cells; lane 2, MGC-803/Silence(-) cells; lane 3, MGC-803/Silence(+) cells. B-actin: internal control protein. (B) Statistical evaluation showed that E2F-1 siRNA significantly reduced E2F-1 protein concentrations in cells.

Psilencer 4.1-E2F-1(+) inhibits cell growth and proliferation in gastric cancer MGC-803 cells. Next, we determined the in vitro survival rates of gastric tumor cells stably transfected with Psilencer 4.1- E2F-1(+) plasmids, using gastric carcinoma cell line MGC-803 as a model for gastric cancer. As shown in Fig. 3, Psilencer 4.1- E2F-1(+) significantly reduced cell survival $(\mathrm{P}<0.05)$, as assessed by MTS assay. MGC-803/Silence(-) cells and MGC-803 cells exhibited 3.3- and 3.2-fold higher mean proliferation rates, respectively than MGC-803/Silence(+) cells. Additionally, we observed that MGC-803/Silence(+) cells obviously grew slower than MGC-803/Silence(-) cells and MGC-803 cells, which was consistent with the decreased levels of E2F-1 in MGC-803/Silence(+) cells and indicated a suppressive effect of Psilencer 4.1- E2F-1(+) on MGC-803 cell growth and survival.

To confirm the inhibitory effect of Psilencer 4.1- E2F-1 (+) on the growth of MGC-803 cells, we performed colony formation assays for measuring the capability of the cell lines to grow in an anchorage-independent environment by culturing the cells in soft agarose. As shown in Fig. 4, three cell lines were able to form colonies in soft agarose but the number of colony formation in MGC-803/Silence(+) cells after three weeks was $46.1 \pm 2.5$, with a 64.2 and $62.3 \%$ decrease, as compared with two control groups, respectively $(\mathrm{P}<0.05)$. Together, these data suggest that E2F-1 siRNA inhibits cell growth and proliferation in the gastric cancer cell system.

Effect of Psilencer 4.1-E2F-1(+) on cell cycle control in gastric cancer MGC-803 cells. We used flow cytometry to determine whether the inhibitory effect of E2F-1 siRNA on
A

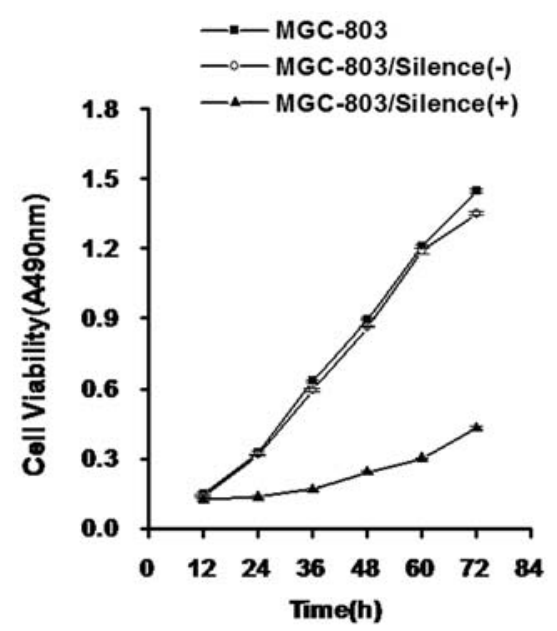

B

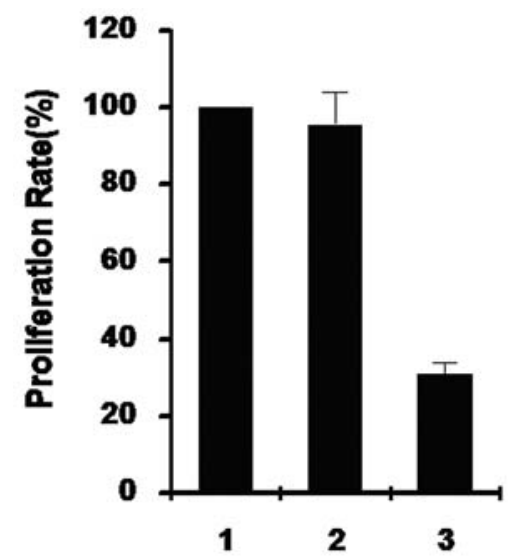

Figure 3. Cell survival assessed by MTS assay. (A) The inhibition of proliferation was observed after MTS treatment. (B) The mean cell proliferation rate of MGC-803/Silence (+) cells was significantly lower. Lane 1, MGC-803 cells; lane 2, MGC-803/Silence(-) cells; lane 3, MGC-803/Silence(+) cells.

Table II. Cell cycle analysis by flow cytometry in gastric cancer cells.

\begin{tabular}{lccc}
\hline \multirow{2}{*}{ Group } & \multicolumn{3}{c}{ Cell cycle phase } \\
\cline { 2 - 4 } & $\mathrm{G}_{0} / \mathrm{G}_{1}(\%)$ & $\mathrm{S}(\%)$ & $\mathrm{G}_{2} / \mathrm{M}(\%)$ \\
\hline MGC-803 cells & 31.9 & 35.1 & 32.9 \\
MGC-803/Silence(-) cells & 36.8 & 29.7 & 33.4 \\
MGC-803/Silence(+) cells & $19.4^{\mathrm{a}}$ & $21.3^{\mathrm{a}}$ & $59.3^{\mathrm{a}}$ \\
\hline
\end{tabular}

a $<0.05$, MGC-803/Silence(+) cells vs. MGC-803 cells and MGC803/Silence(-) cells.

MGC-803 cell proliferation was mediated, at least in part, through affecting cell cycle progression. We found that MGC-803/Silence (+) cells were $19.4 \%$ at $\mathrm{G}_{0} / \mathrm{G}_{1}$ phase and $59.3 \%$ at $\mathrm{G}_{2} / \mathrm{M}$ phase, with 80.2 and $77.5 \%$ increase in $\mathrm{G}_{2} / \mathrm{M}$ phase cell population and 39.2 and $47.3 \%$ decrease in $\mathrm{G}_{0} / \mathrm{G}_{1}$ phase cell population, as compared to MGC-803 cells and MGC-803/Silence(-) cells ( $\mathrm{P}<0.05)$ (Table II and Fig. 5). These data indicate that cell growth inhibition by Psilencer 4.1- 
A

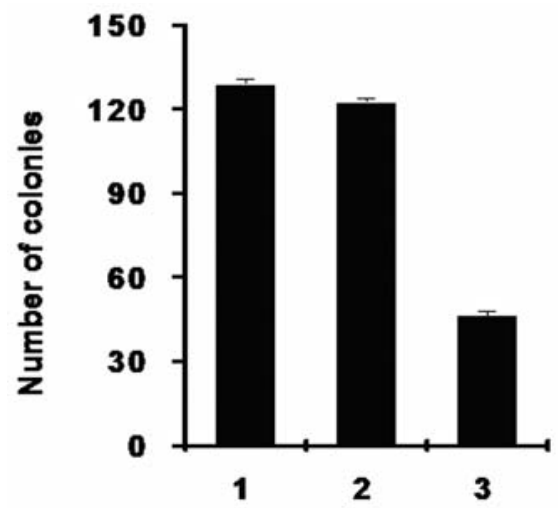

B
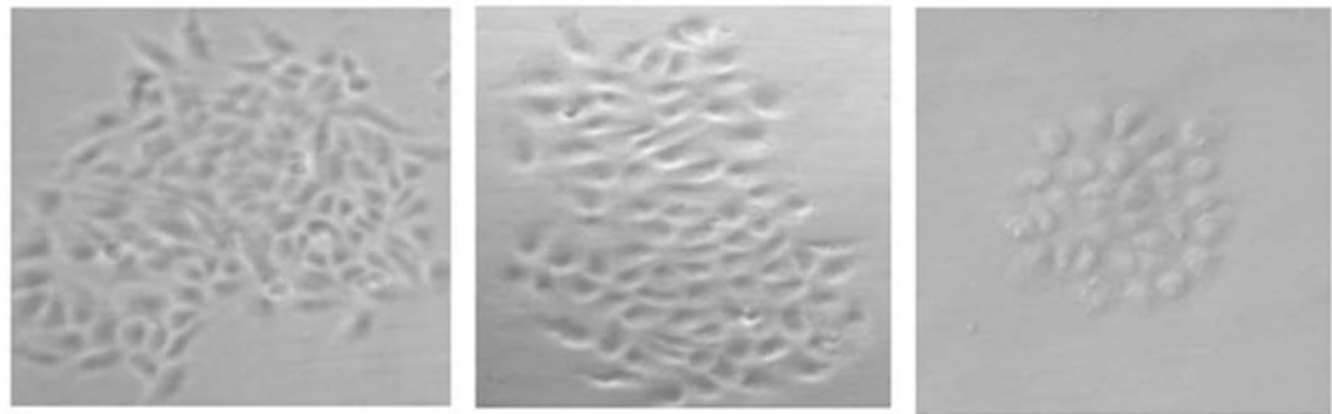

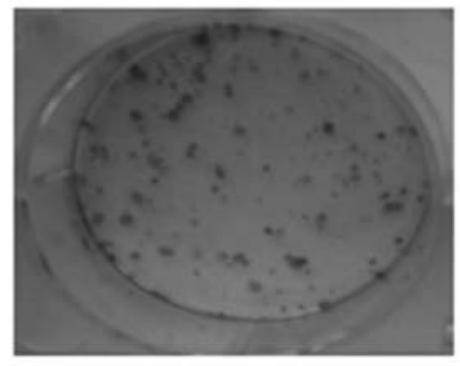

1

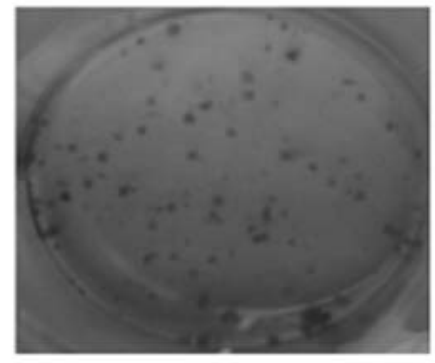

2

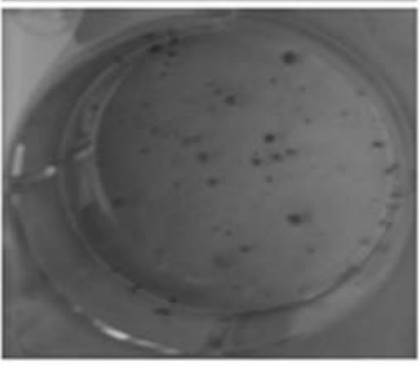

3

Figure 4. Results of colony formation assay. (A) MGC-803/Silence(+) cells exhibited fewer colonies than MGC-803/Silence(-) cells or MGC-803 cells. (B) A representative result of the colony formation assay. Lane 1, MGC-803 cells; lane 2, MGC-803/Silence(-) cells; lane 3, MGC-803/ Silence(+) cells.

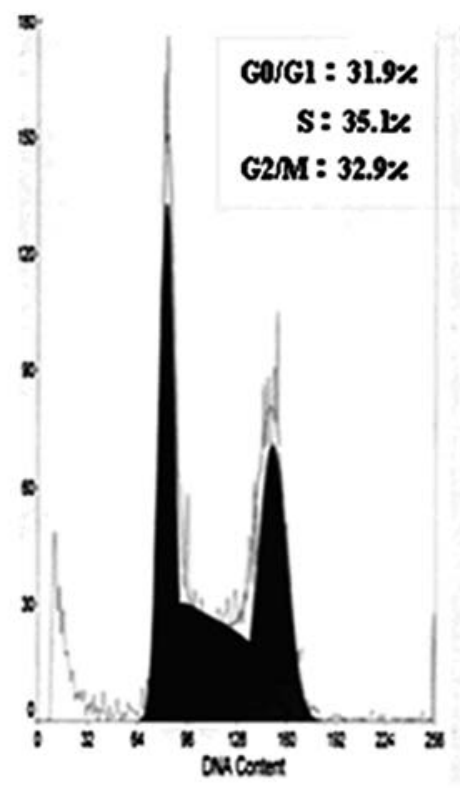

1

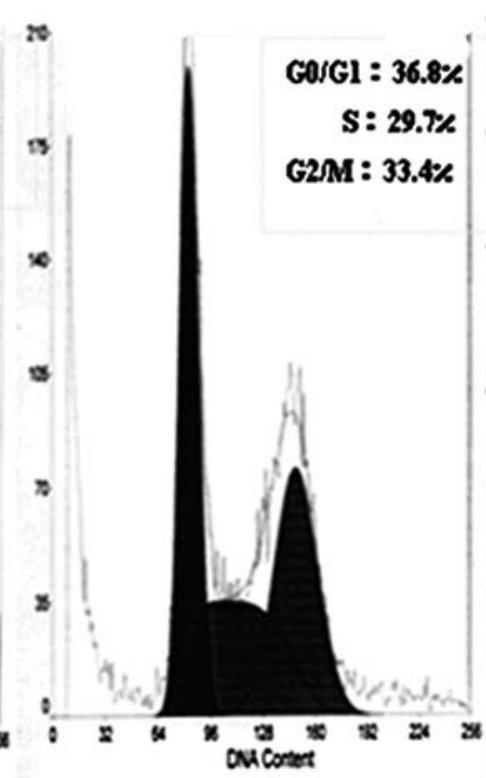

2
GOJG1 : 19.4\%

S: 21.34 G2M : 59.3\%

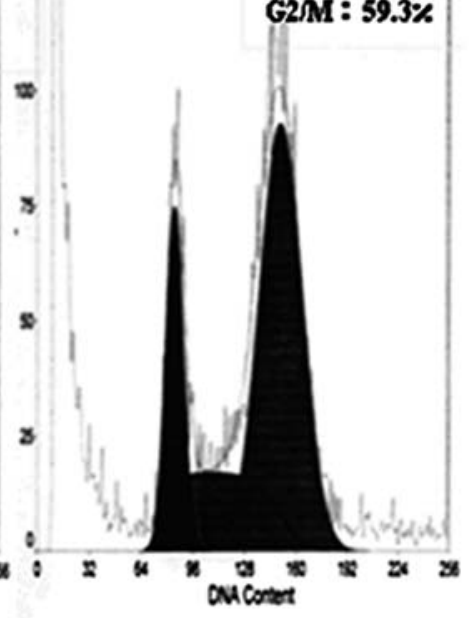

3

Figure 5. Cell cycle analysis by flow cytometry. Lane 1, MGC-803 cells; lane 2, MGC-803/Silence(-) cells; lane 3, MGC-803/Silence(+) cells. 

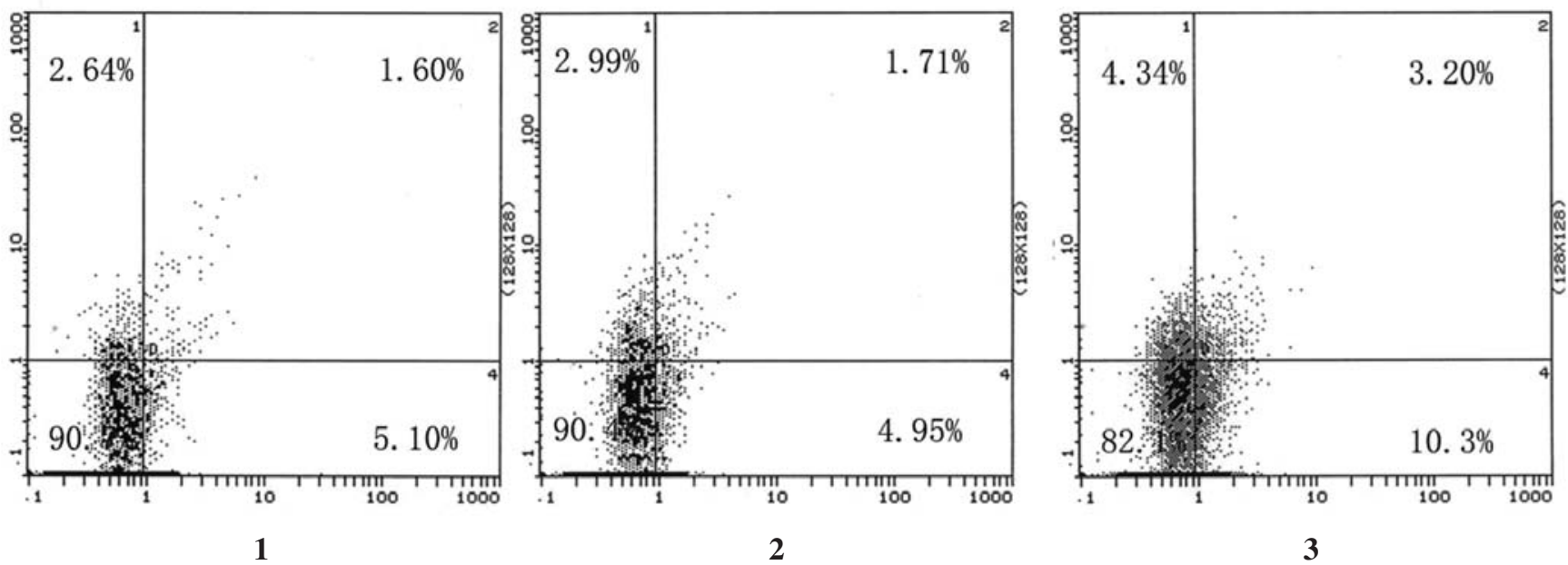

Figure 6. Percentages of apoptotic cells analyzed by flow cytometry. Lane 1, MGC-803 cells; lane 2, MGC-803/Silence(-) cells; lane 3, MGC-803/Silence(+) cells.

A

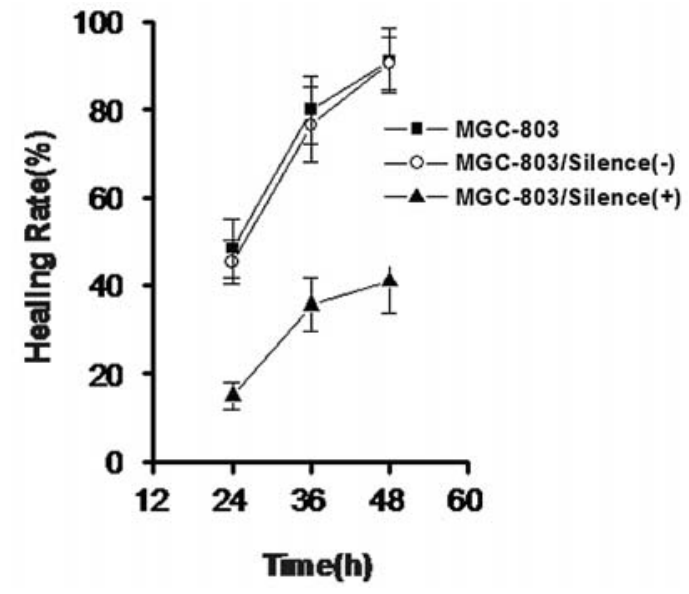

E2F-1(+) is associated with significant cell cycle arrest in $\mathrm{G}_{2} / \mathrm{M}$ phase and suggest that siRNA directed against the E2F-1 gene suppresses cell proliferation by controlling the $\mathrm{G}_{2} / \mathrm{M}$ checkpoint and inducing a specific block in cell cycle progression.

Psilencer 4.1-E2F-1(+) transfection induces cellular apoptosis. To further study the effect of E2F-1 on MGC-803 cell apoptosis, cells were stained with Annexin V-FITC and PI, and then subsequently analyzed by flow cytometry. This assay was based on the translocation of phosphatidylserine from the inner leaflet of the plasma membrane to the cell surface in the early apoptotic cells. The dual parameter

B
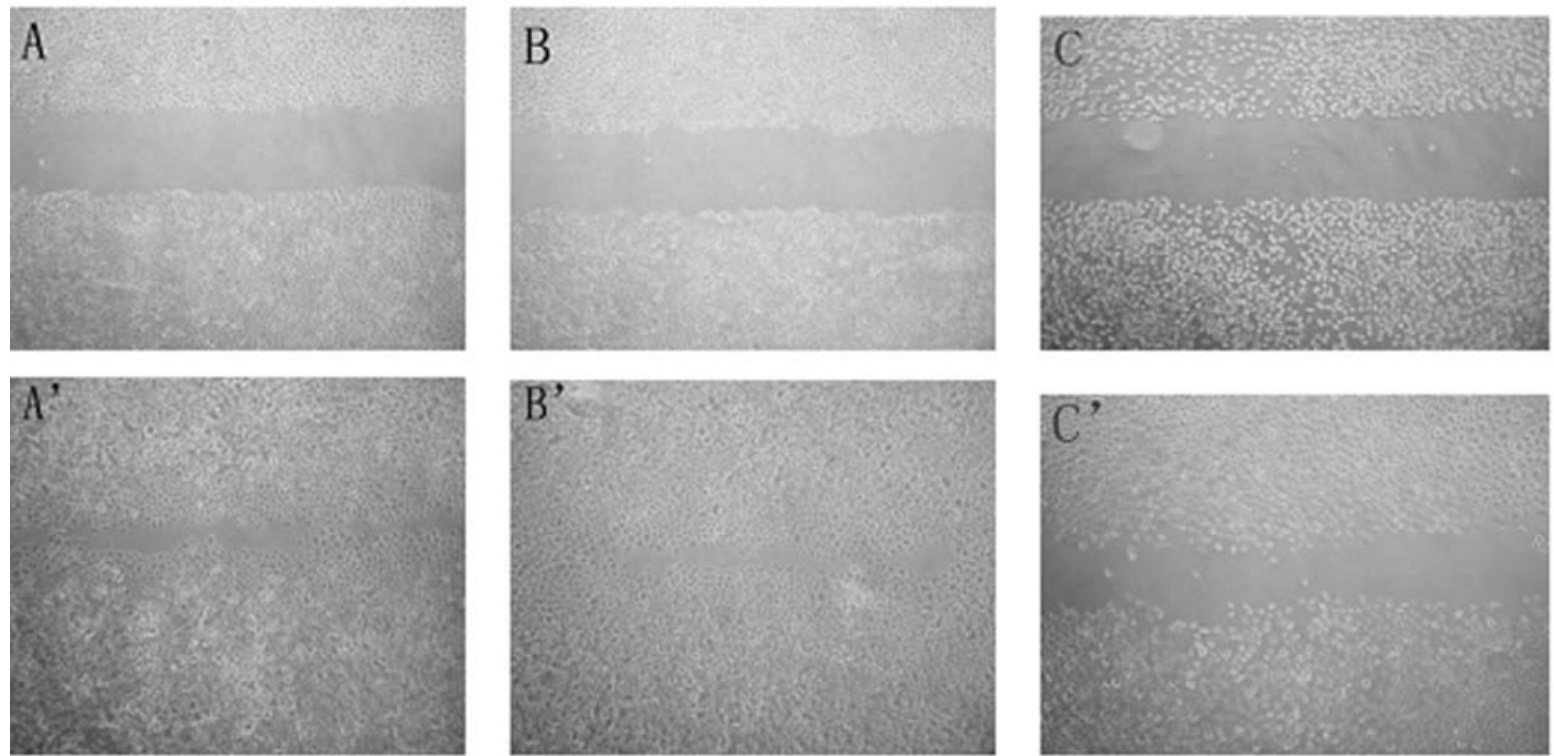

Figure 7. (A) E2F-1 siRNA decreased migration of MGC-803 cells in wound healing assay. (B) A representative result of cell migration. (A) MGC-803 cells at $0 \mathrm{~h}$; (A') MGC-803 cells at 48 h; (B) MGC-803/Silence(-) cells at 0 h; (B') MGC-803/Silence(-) cells at 48 h; (C) MGC-803/Silence(+) cells at $0 \mathrm{~h} ;\left(\mathrm{C}^{\prime}\right)$ MGC-803/Silence $(+)$ cells at $48 \mathrm{~h}$. 
fluorescent dot plots showed that the viable cells were in the lower left quadrant (Annexin V-/PI-), the cells at the early apoptosis were in the lower right quadrant (Annexin V+/PI-), and the ones at the late apoptosis or necrosis were in the upper right quadrant (Annexin $\mathrm{V}+\mathrm{PI}+$ ). As indicated in Fig. 6, the percentage of MGC-803/Silence(+) cells with Annexin V+/ PI- was significantly higher than that of MGC-803/Silence(-) or MGC-803 cells $(\mathrm{P}<0.05)$. It implies that inhibition of E2F-1 was able to induce apoptosis in gastric cancer MGC803 cells.

Psilencer 4.1-E2F-1(+) transfection decreases migration and invasion of gastric cancer cells. We measured the migration ability of three cell groups using the wound healing assay by scratching the single-layer cells. The distance between the wound edges was determined at 0, 24, 36 and $48 \mathrm{~h}$ and the healing rate was calculated in the three groups. MGC-803/Silence(+) cells showed a lower migration ability at the time-points evaluated than MGC-803/Silence(-) cells $(\mathrm{P}<0.05)$ and MGC-803 cells $(\mathrm{P}<0.05)$. The healing rate of MGC-803/ Silence(+) cells after 48 h was $40.9 \pm 7.2 \%$, with 54.8 and $55.1 \%$ decrease, as compared with MGC803/Silence(-) cells and MGC-803 cells (Fig. 7).

Because siRNA targeting of E2F-1 inhibited the expression of E2F-1 gene in gastric cancer cells, we assessed its ability to inhibit cell invasion. After incubation for $24 \mathrm{~h}$ in the invasion assay, the numbers of MGC-803/Silence(-) and MGC-803 cells invaded through the membrane of Matrigel chamber were 2.7- and 3.0-fold greater than that

A

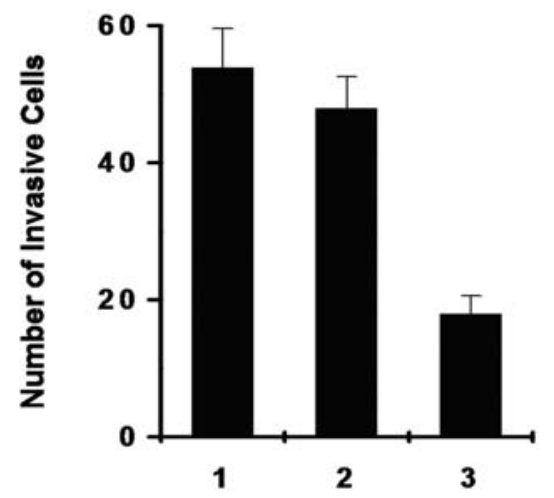

of MGC-803/Silence(+) cells, respectively $(\mathrm{P}<0.05)$ (Fig. 8). The results indicate that E2F-1 siRNA reduces migration and invasion ability of gastric cancer MGC- 803 cells.

\section{Discussion}

Gastric cancer is a worldwide problem. Besides the undetermined etiological factors, there are also limitations in surgery, chemotherapy and radiotherapy, the major therapies for gastric cancer to date (13). Many patients lose the chance of surgery because of their systemic condition, while many cannot tolerate the side effects of chemotherapy or radiotherapy. It is important to find a new way to effectively inhibit cancer cell growth and avoid the side effects of drugs or radioactive rays. Gene target therapies have proved to be a promising way to achieve this goal.

The recent discovery of RNAi, is a strong tool for silencing the function of specific genes, compared with the conventional gene-suppression technologies such as chemical inhibitors, antisense technology, ribozymes and deoxyribozymes, which often evoke non-specific side effects and/or offer only transient and partial suppression of the gene of interest. RNAi is a more potent inhibitor of gene expression with less toxicity (14). More importantly, given the potency and effectiveness of RNAi as a gene silencing tool, RNAi has significant therapeutic potential for human applications. Thus, RNAi-mediated gene silencing has opened new avenues in cancer therapy. The feasibility of RNAi-mediated gene silencing as a novel tool to arrest tumor growth and kill cancer cells is being tested in several laboratories and their initial results are promising (15).

In the present study, we constructed recombinant Psilencer 4.1-E2F-1 siRNA plasmids and successfully transfected these plasmids into gastric cancer MGC-803 cells. Our results clearly showed that the transfection of Psilencer 4.1E2F-1(+) plasmid led to remarkable inhibition of E2F-1 mRNA and protein expression in MGC-803 cells, and it inhibited cell growth and made cell cycle arrest in $\mathrm{G}_{2} / \mathrm{M}$ phase. The results in our study also showed that E2F-1 siRNA induced cell apoptosis. Furthermore, RNAi-directed targeting
B

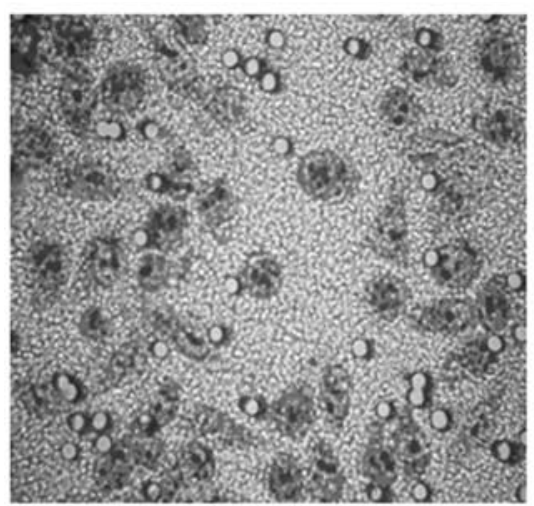

1

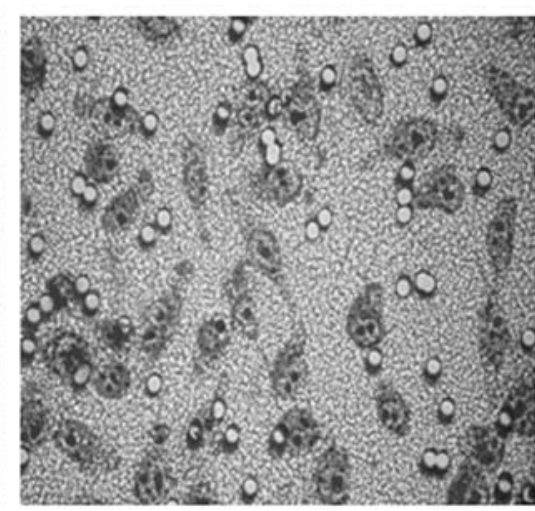

2

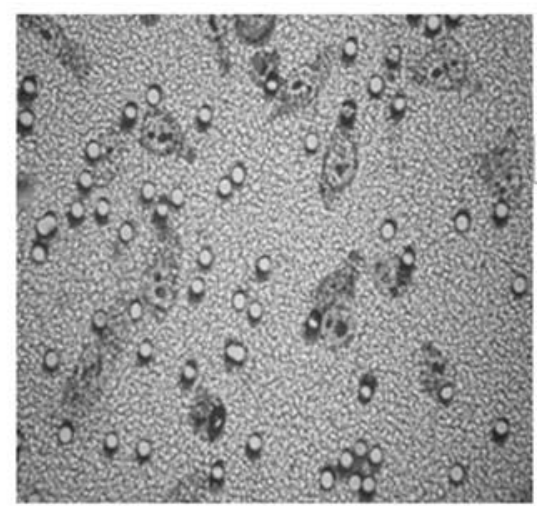

3

Figure 8. (A) E2F-1 siRNA decreased invasion of MGC-803 cells. (B) A representative result of cell invasiveness obtained from three independent experiments. Lane 1, MGC-803 cells; lane 2, MGC-803/Silence(-) cells; lane 3, MGC-803/Silence(+) cells. 
of E2F-1 in MGC-803 cells could reduce the capability of cell motion, invasion and colony formation. The above evidence indicates that siRNA targeting E2F-1 plays an important role in the inhibition of gastric cancer progression in vitro. These inhibitory effects were due to posttranscriptional silence mechanisms, in which the synthetic, double-stranded siRNA effectively silenced E2F-1 in gastric cancer MGC-803 cells. This is consistent with many studies, which showed that the introduction of a $21 \mathrm{nt}$ dsRNA into cancer cells strongly suppressed the expression of specific mRNAs $(16,17)$. The sequence of this dsRNA was highly homologous and unique to a region of the targeted mRNA (18). The expression of E2F-1 protein was therefore inhibited, and the percentage of apoptotic cells was higher than that of control groups and the tumor growth was obviously suppressed.

The E2F transcription factor can regulate expression of numerous cellular genes controlling proliferation, including proto-oncogenes and genes regulating cell cycle progression. Therefore, the genes comprising the E2F gene family could potentially contribute to carcinogenesis. E2F-1 was the first cloned and is the better characterized member of this gene family. E2F-1 is the best-known transcription factor regulated by the cyclin-Cdk-Rb pathway. E2F-1 controls the expression of genes encoding various DNA replication proteins and cell cycle regulators, and it seems logical that E2F-1-dependent expression of these genes would be needed to sustain cell proliferation $(19,20)$.

Previous observations indicated that inhibition of E2F-1 activity led to a perturbation of cell cycle progression. Thus, an E2F-1 dominant-negative mutant lacking the DP-binding site inhibited both the transcriptional activity of E2F-1 and cell proliferation (21). Likewise, a dominant-negative DP-1 mutant that forms inactive heterodimers with E2F proteins has been reported to induce a cell cycle arrest in $G_{1}$, and RNA aptamers that specifically blocked E2F DNA binding also inhibited cell proliferation after microinjection into primary fibroblasts $(22,23)$. Our work confirms and extends these observations. In the present study, we reported the inhibitory effects of specific siRNA on E2F-1 expressed in human gastric cancer cells and the results showed that E2F-1 siRNA was able to significantly reduce cell growth and induced a cell cycle arrest in $\mathrm{G}_{2} / \mathrm{M}$.

Apart from its essential function in cell cycle progression, a role for E2F-1 in regulating apoptosis is well documented. The physiological relevance of E2F-1 control in promoting cell death has been clearly demonstrated by the development of spontaneous tumors in E2F-1 null mice, indicating that E2F-1 can act as a tumor suppressor gene (24). This function of E2F-1 is in part because of its ability to activate proapoptotic genes, such as p73 and Apaf-1 $(25,26)$. The observations made in the present study are in apparent contrast to this proapoptotic function of $\mathrm{E} 2 \mathrm{~F}-1$, but is in line with several other reports in the literature. A short DP-1-derived peptide linked to penetratin to enable cellular translocation inhibited E2F-DP heterodimerization and caused apoptosis of tumor cell lines (27). It has been described that E2F activity is essential for the survival of Myc-overexpressing tumor cells regardless of their ARF and p53 status (28). It would thus appear that E2F-1 can exert opposing functions with respect to the regulation of apoptosis, and that the precise outcome of E2F-1 inhibition may depend on the genetic status of the cell and the loss-of-function approach.

One characteristic feature of any malignant tumor is the ability of tumor cells to invade and migrate into surrounding and/or distal tissue. Cell invasion is due to degradation of the ECM and the basement membrane surrounding the primary tumor by proteases. These proteases are involved in tumor growth and invasion at both primary and metastatic sites, particularly at the invading foci. Although several extracellular protease systems have been implicated in the tissue degradation and remodeling that often accompanies cancer invasion, several studies demonstrated that the E2F-1 system is important to these processes (29). In the present study, we found inhibition of migration and invasion potential of MGC-803 cells in vitro because of the transfection of Psilencer 4.1- E2F-1(+) plasmid.

Our previous study showed that E2F-1 overexpression in gastric cancer MKN-45 cells produced similar results to E2F-1 siRNA (30). However, the inhibition by E2F-1 overexpression was associated with cell cycle arrest in $\mathrm{G}_{1} / \mathrm{S}$ phase but not $\mathrm{G}_{2} / \mathrm{M}$ phase as $\mathrm{E} 2 \mathrm{~F}-1$ siRNA. It suggests that appropriate activity and expression level of E2F-1 are necessary for normal cell cycle, even in promoting tumor proliferation and regression. Upregulation or downregulation of E2F-1 can suppress gastric cancer progression in vitro but with different mechanisms.

In conclusion, the downregulation of E2F-1 using RNAi successfully reduced the proliferation, migration and invasion of MGC-803 cells in vitro. These findings suggest that RNAi-directed targeting of E2F-1 can be used as a potent and specific therapeutic tool for the treatment of gastric cancer, especially in inhibiting and/or preventing cancer cell progression.

\section{Acknowledgements}

This work was supported by grants from the Natural Science Foundation of China (No. 30860273), the Natural Science Foundation of Guangxi (No. 0640085) and the Key Health Science Foundation of Guangxi (No. Key 200844), China.

\section{References}

1. Yang L: Incidence and mortality of gastric cancer in China. World J Gastroenterol 12: 17-20, 2006.

2. Weinberg RA: The retinoblastoma protein and cell cycle control. Cell 81: 323-330, 1995.

3. Bartek J, Bartkova J and Lukas J: The retinoblastoma protein pathway and the restriction point. Curr Opin Cell Biol 8: 805-814, 1996.

4. Sherr CJ: Cancer cell cycles. Science 274: 1672-1677, 1996.

5. Sherr CJ: The Pezcoller lecture: cancer cell cycles revisited. Cancer Res 60: 3689-3695, 2000.

6. Johnson DG, Cress WD, Jakoi L and Nevins JR: Oncogenic capacity of the E2F1 gene. Proc Natl Acad Sci USA 91: 12823-12827, 1994.

7. Suzuki T, Yasui W, Yokozaki H, Naka K, Ishikawa T and Tahara E: Expression of the E2F family in human gastrointestinal carcinomas. Int J Cancer 81: 535-538, 1999.

8. Rabbani F, Richon VM, Orlow I, et al: Prognostic significance of transcription factor E2F-1 in bladder cancer: genotypic and phenotypic characterization. J Natl Cancer Inst 91: 874-881, 1999.

9. Muller $\mathrm{H}$ and Helin K: The E2F transcription factors: key regulators of cell proliferation. Biochim Biophys Acta 1470: M1-M12, 2000. 
10. Zhang SY, Liu SC, Johnson DG and Klein-Szanto AJ: E2F-1 Gene transfer enhances invasiveness of human head and neck carcinoma cell lines. Cancer Res 60: 5972-5976, 2000

11. Zamore PD: RNA interference: listening to the sound of silence. Nat Struct Biol 8: 746-750, 2001.

12. Hammond SM, Bernstein E, Beach D and Hannon GJ: An RNA-directed nuclease mediates post-transcriptional gene silencing in Drosophila cells. Nature 404: 293-296, 2000.

13. Cunningham D, Jost LM, Purkalne G and Oliveira J: ESMO minimum clinical recommendations for diagnosis, treatment and follow-up of gastric cancer. Ann Oncol 16 (Suppl. 1): i22-i23, 2005.

14. McCaffrey AP, Meuse L, Pham TT, Conklin DS, Hannon GJ and Kay MA: RNA interference in adult mice. Nature 418: 38-39, 2002.

15. Wilda M, Fuchs U, Wossmann W and Borkhardt A: Killing of leukemic cells with a BCR/ABL fusion gene by RNA interference (RNAi). Oncogene 21: 5716-5724, 2002.

16. Cerutti H: RNA interference: traveling in the cell and gaining functions? Trends Genet 19: 39-46, 2003.

17. Dykxhoorn DM, Novina CD and Sharp PA: Killing the messenger: short RNAs that silence gene expression. Nature Rev Mol Cell Biol 4: 457-467, 2003.

18. Coumoul X, Li W, Wang RH and Deng C: Inducible suppression of Fgfr2 and Survivin in ES cells using a combination of the RNA interference (RNAi) and the Cre-LoxP system. Nucleic Acids Res 32: 85, 2004.

19. Nevins JR: The Rb/E2F pathway and cancer. Hum Mol Genet 10: 699-703, 2001.

20. Johnson DG, Schwarz JK, Cress WD and Nevins JR: Expression of transcription factor E2F1 induces quiescent cells to enter S phase. Nature 365: 349-352, 1993.
21. Fan $\mathrm{J}$ and Bertino JR: Functional roles of E2F in cell cycle regulation. Oncogene 14: 1191-1200, 1997.

22. Wu CL, Classon M, Dyson N and Harlow E: Expression of dominant-negative mutant DP-1 blocks cell cycle progression in G1. Mol Cell Biol 16: 3698-3706, 1996.

23. Ishizaki J, Nevins JR and Sullenger BA: Inhibition of cell proliferation by an RNA ligand that selectively blocks E2F function. Nat Med 2: 1386-1389, 1996.

24. Field SJ, Tsai FY, Kuo F, et al: E2F-1 functions in mice to promote apoptosis and suppress proliferation. Cell 85: 549-561, 1996.

25. Irwin M, Marin MC, Phillips AC, et al: Role for the p53 homologue p73 in E2F-1-induced apoptosis. Nature 407: 645-648, 2000.

26. Furukawa Y, Sutheesophon K, Wada T, Nishimura M Saito Y, Ishii $\mathrm{H}$ and Furukawa $\mathrm{Y}$ : Apaf-1 is a mediator of E2F-1-induced apoptosis. J Biol Chem 277: 39760-39768, 2002.

27. Silvia M, Rolf M and Roland EK: Inhibition of cell proliferation and induction of apoptosis by novel tetravalent peptides inhibiting DNA binding of E2F. Oncogene 22: 4943-4952, 2003.

28. Santoni-Rugiu E, Duro D, Farkas T, Mathiasen IS, Jaattela M, Bartek J and Lukas J: E2F activity is essential for survival of Myc-overexpressing human cancer cells. Oncogene 21: 6498-6509, 2002.

29. Xiao Q, Li L, Xie Y, et al: Transcription factor E2F-1 is upregulated in human gastric cancer tissues and its overexpression suppresses gastric tumor cell proliferation. Cell Oncol 4: 335-349, 2007. 